\title{
Characterization of Flex to Printed Circuit Board Interconnections using Insertion Connectors
}

\author{
Ch. Person ${ }^{\#}$, Duc NGuyen ${ }^{\#}$, J.Ph. Coupez ${ }^{\#}$, Ph. Minard*, D. Lo Hine Tong*, P. Borel ${ }^{\S}$, D. Izoard ${ }^{\S}$ \\ ${ }^{*}$ LabSTICC, IMT Atlantique, France \\ ${ }^{*}$ Technicolor Connected Home, France \\ $\S_{\text {Centre Technique du Papier, France }}$ \\ \#\{christian.person, hong.nguyen, jp.coupez\}@imt-atlantique.fr, ${ }^{2}$ \{philippe.minard, dominique.lohinetong@technicolor.com\}, \\ ${ }^{3}$ Pascal.Borel, damien.izoard@webctp.com\}
}

\begin{abstract}
Advanced internet and multimedia set-top boxes are today massively based on $4 * 4$ MIMO systems, with multisub-bands standards (WIFI bands, LTE, Bluetooth,...). Interconnections between the numerous access ports of different radio chipsets mounted on a main PCB (Printed Circuit Board) and multiple antennas spatially distributed on the plastic casing become therefore a great challenge for cost and performances motivations. The flex technology, based on well-controlled and low cost process, brings new functionalities and opportunities for assembling sub-systems, under compactness considerations, as well as electrical performances improvement.

In this paper, we discuss about the characterisation of connectors used for flexible printed circuit to a main PCB interconnections. The choice of the connectors is a critical issue, and must be properly done considering both local ground and signal interconnections constraints. Simulation and measurements are performed up to $15 \mathrm{Ghz}$ covering WIFI bands.
\end{abstract}

Keywords -- RF interconnection, Flexible Printed Circuit, paper substrates, conformable structure

\section{INTRODUCTION}

In commercial electronics devices, different interfaces need to be interconnected. Therefore, RF antennas, as well as batteries, or LCD displays for instance, are usually fixed to the housing box of the equipment, and interfaced with the central processing electronic unit though cables and miniaturized multiple input-output connectors.

Even if such techniques are not really low cost, they offer undoubtedly flexibility in terms of test, repairment, RF efficiency. They are still considered in advanced electronic devices as required functionnalities tend to promote the integration of various wireless modules in a common environment to cover various design features and functionnalities (cohabitation of different standards, necessitating various antennas). In addition, antennas are usually processed on substrates external to the main PCB, so as to address specific constraints such as radiation efficiency (thus using low-loss cost effective thin substrates), spatial diversity for MIMO schemes (then requiring up to $4 * 4$ colocated antennas per standards and multiple interconnections towards the main PCB).
Commercial wireless systems have consequently strongly encouraged the development of a new familiy of miniaturized RF connectors and cables, with particular expectations in terms of fast and accurate mounting techniques as well as mass-production for cost-reduction.

The project "Stick'It" ("Printable passive antenna system a a conformable structure") aims to develop innovative low-cost technologies for the design of $2 \mathrm{D}$ and $2.5 \mathrm{D}$ RF interconnection components and sub-systems printed on flexible materials such as paper substrates and plastic films. We focus on the research for interconnection solutions between sub-systems made partly on flexible supports. The final objective consists in replacing conventionally used solutions (coaxial cables + connectors) by low-cost flex to PCB direct connections.

In this paper, Part II rapidly describes the conventional solutions used for interconnecting RF antennas and main board through miniaturized cables and connectors. Part. III is dedicated to studies carried out to identify connector solutions compatible with the constraints of flex technologies (paper-based or polymer-based substrates) The last Part presents experimentations performed with the selected connectors for flex paper substrate to main PCBs antenna connections.

\section{NEW INTERCONNECTION CONSTRAINTS WITH FLEXIBLE ELECTRONIC COMPONENTS}

\section{A. E4D Paper as a flexible support for conformable $R F$ devices implementation}

We have tested different metallization methods and cellulosic based substrates for evaluating a new family of ultra-low cost interconnection substrates. Ag-screen printed lines on both side of E4D papers have been designed and fully characterized up to $6 \mathrm{GHz}$. Microstrip transmission line have been screen-printed using silver ink ( $7 \mu \mathrm{m}$ thickness) on a 240 $\mu \mathrm{m}$ thick E4D paper substrate (experimental dielectric properties: $\varepsilon \mathrm{r}=2.54$ and $\tan \delta=0.05)[1]$. 
The surface roughness of the paper substrate and the printed patterns have been identified as key parameters. Insertion loss of about $1.73 \mathrm{~dB} / \mathrm{cm}$ for screen-printed line on E4D paper at $20 \mathrm{GHz}$ but we obtained quite acceptable performances for antennas and interconnection lines within the conventional WIFI frequencies ranges.

The development of such substrates will make paper, in the near future, an excellent candidate for ultra-low-cost, flexible, and environmental friendly electronics. We thus investigated on the next issue: interfacing devices printed on ultra low-cost flex materials (paper and polymers substrates) with a centre PCB (Printed circuit Board) based on a rigid conventional material.

\section{B. Conventional interconnections through cables and plug-in connectors}

The interconnection solution which consists in using coaxial cable is expensive in terms of implementation cost, so we have investigated for a new ultra-low-cost interconnection solution on paper and flex. The antennas and their interconnection lines are printed on the same paper sheet. Therefore, the last technological breakthrough is the direct interconnection between the flexible feed lines and the main PCB.

Commercial products such as RF surface mounted microconnectors have been developped for such purpose, with Miniature Plug in connectors. In addition, original techniques have been studied to facilitate plug-in and plug-out operations, as well as elastic/flexible board to board clipping or contact solutions to counteract eventual damages related to temperature variations or mechanical shocks.

For RF antennas, micro-cables are usually and preferably directly soldered and connected to the input port, in order to minimize cost and to optimize impedance matching and ground plane continuity. Central pin and shielding structures are usually soldered to the main $\mathrm{PCB}$, considering microstrip configurations (top main line + inner ground plane). This requires usually via holes to promote surface soldered cables, as described on figure 1 below.

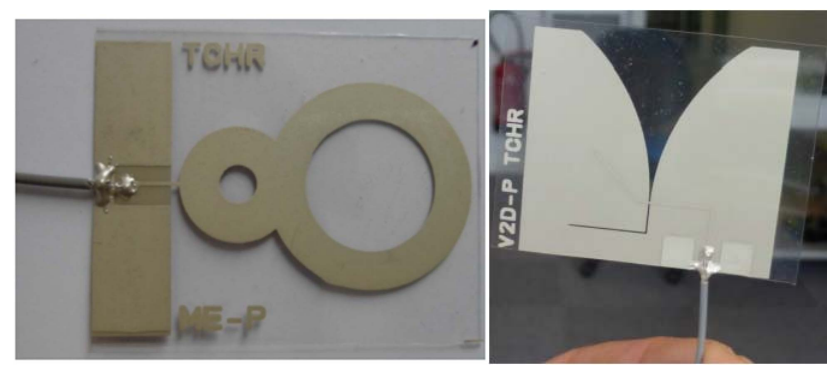

Fig. 1. View of a coaxial interconnection directly soldered to the PCB (courtesy of Technicolor Connected Home Company)
Such solution brings several drawbacks for mass production:

- A recurrent cost (especially when higher frequencies (above 2Ghz) are considered:

- Non-repairable systems: Cables are soldered, with consequently difficulties to repair and disconnect the attached PCBs

- Difficulties to control undesired coupling and radiating phenomena (EMC constraints)

- Space occupation, especially when multiple antennas systems are considered, thus necessitating to keep sufficient place for soldering operations.

- Some potential mismatching depending on the quality of the soldering operation

\section{INTERCONNECTION BETWEEN FLEXIBLE PRINTED CIRCUIT AND A MAIN PRINTED CIRCUIT BOARD}

\section{A. Identifying a connector compatible with RF interconnects}

Considering previous drawbacks, we target to identify original interconnection techniques/systems to propose multi-access miniature interfaces between a flex support and a main PCB board. This leads to emerging concepts of Flexible Printed Circuit (FPC) and Flexible Flat Cable (FFC) interfaces.

Numerous types of connectors are available on the market for FPC to PCB connections, with different mechanical and electrical expectations (robustness, supported currents and powers, operational frequencies and data bit rate, tolerances and achievable dimensions, costs....). In addition, numerous parameters appear as critical issues at RF frequencies for guaranteeing the control of the signal continuity (main signal and ground planes). In particular :

- Availability of one face or double face contacts

- Number of contact pins and associated spacing/width

- Presence of a lock system to maintain the flex in closed contact with the metallic pins

- Height of the aperture for flex insertion $(200 \mu \mathrm{m}$ to $1 \mathrm{~mm})$.

Various manufacturers, like Hirose ${ }^{\mathrm{TM}}$, Molex ${ }^{\mathrm{TM}}$, Amphenol $\mathrm{FCi}^{\mathrm{TM}}$, TE Connectivity ${ }^{\mathrm{TM}}$, Goldenconn ${ }^{\mathrm{TM}}$, etc..., offer diverse configurations, usually inspired from digital high data bit rate multi-pins connectors families. We have selected connectors fabricated by Hirose ${ }^{\mathrm{TM}}$, considering advantages in terms of compactness, quality and the certified performance of the interconnections and the insertion technique of the flexible substrate compatible with paper characteristics (Zero Insertion Force ZIF connectors).

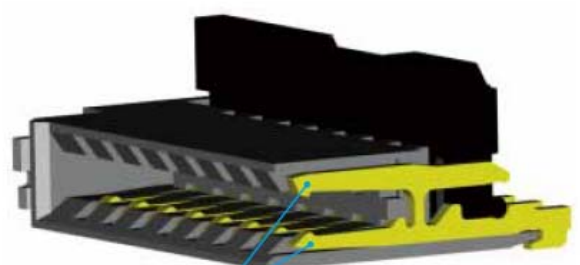

Fig. 2. Perspective view of a SMC type ZIF connector (Hirose ${ }^{\mathcal{O}}$ ). 
The selection criteria here corresponds to the dimensions of the aperture, fully compatible with thin $(200 \mu \mathrm{m}$ thick substrates) and the dimensions of the miniaturized top of bottom pins for local ground plane continuity.

\section{B. Preliminary RF Characterisation}

In order to verify the compatibility of these connectors for radio frequency operations, two $50 \Omega$ microstrip transmission lines have been tested, with interconnection through one ZIF connector. The idea herein is that: one line is on a rigid substrate (representing the PCB main board); the other line is on the flexible substrate (representing flexible feed lines of the antenna system).

A preliminary test has been done considering two $50 \Omega$ microstrip transmission lines realized on the same flexible substrate (Teflon ${ }^{\mathrm{TM}}$ substrate (thickness $250 \mu \mathrm{m}, \varepsilon_{\mathrm{r}}=2.2, \tan \delta$ $=0.0003)$ ): one was soldered with a surface mounted ZIF connector; the other can be inserted into the opening of this connector to form an interconnection. [2]

This substrate was chosen thanks to its flexibility and its low loss tangent. The $250 \mu \mathrm{m}$ thickness was also selected to be compatible with the connector dimensions including the $17 \mu \mathrm{m}$ thick copper metallization.

Two possible configurations have been developed: (a) contact on only one side of the flex line combining with vias realization and (b) contact on the both sides of the flex line. The vias were realized manually in this trial run.

\section{1) Configuration 1 : Contacts on the top side of the flex lines}

The FH34SJ-4S connector was used for this configuration. It has 4 contacts only on the top of the flex line. So, 2 contact tracks in the middle are used for the signal line and 2 contact tracks at the edges are used for the ground plane. The design for the interconnection is presented in figure 3 .

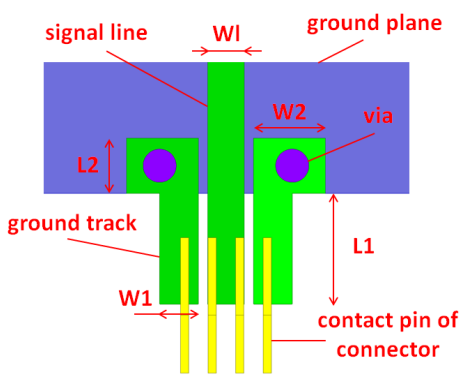

Flexible line
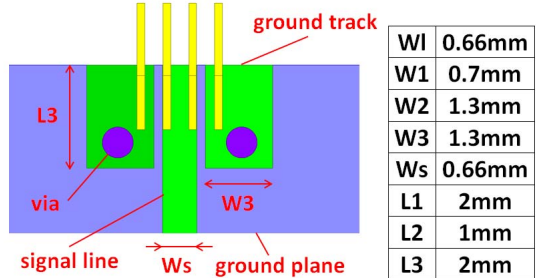

On the PCB side

Fig. 3. Design for the interconnection in the case of using connector with contacts on top side of the flex line.
The simulation model on HFSSTM is illustrated on figure 4. A simplified model was established do describe the complex connector structure, considering only metallic parts, neglecting therefore complex plastic forms.

Each transmission line has a length of $30 \mathrm{~mm}$ and a width of $0.66 \mathrm{~mm}$ corresponding to a $50 \Omega$ impedance. Two vias must be realized on the flex line in order to have contact with the ground plane.

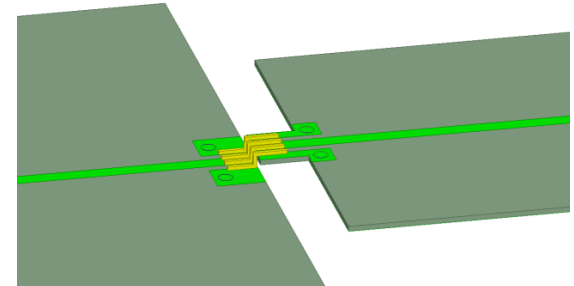

(a)

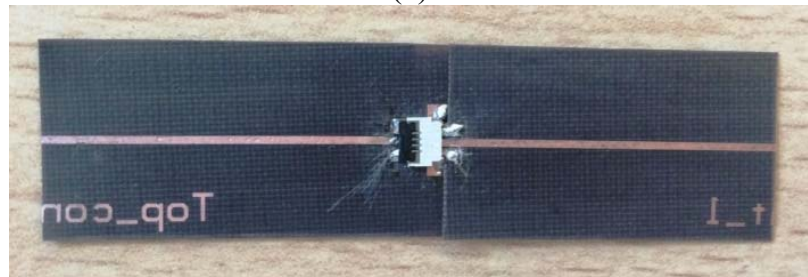

(b)

Fig. 4. Simulation model (a) and prototype (b) of the interconnection using connector with contacts on top side of the flex line

The performance of these two transmission lines through the tested connector are presented in figure 5. The measurement is not totally smooth due to resonances between the two edges of the metallic support with the two ground planes of the two microstrip transmission lines. However, the agreement with the simulation results remains fairly good. The measured $\mathrm{S}_{21}$ exhibits additional losses than simulation, due to extra losses of the two connectors not included in the ideal simulation (dielectric losses of the connector housing material neglected). In addition, via holes have been realized manually with some imperfections for the connections to ground plane.

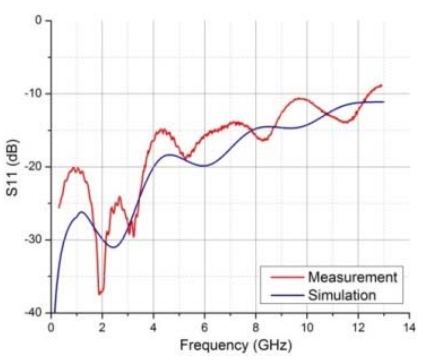

Return loss

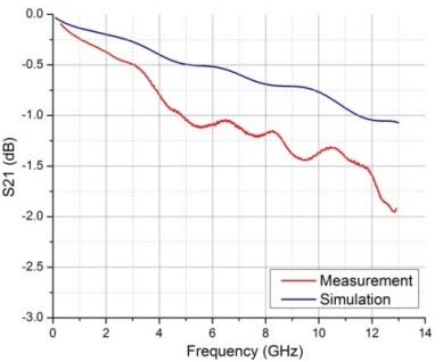

Insertion loss
Fig. 5. $\mathrm{S}_{\mathrm{ij}}$ parameters of the transmission line through ZIF connector having contacts on top side of the flex line

We can notice that the impedance matching is very good for frequencies below $3 \mathrm{GHz}$, and it is gradually degraded by rising in higher frequencies. The insertion losses over the $6 \mathrm{~cm}$ length of two transmission lines through the connector were about $-0.5 \mathrm{~dB}$ at $2.4 \mathrm{GHz}$ and $-1.1 \mathrm{~dB}$ at $5.5 \mathrm{GHz}$. 
Considering the insertion losses of a Teflon ${ }^{\mathrm{TM}} 50 \Omega$ transmission line $(0.27 \mathrm{~dB}$ at $2.4 \mathrm{GHz}$ and $0.47 \mathrm{~dB}$ at 5.5 $\mathrm{GHz}$ ), the insertion losses through the connector are estimated to about $0.23 \mathrm{~dB}$ at $2.4 \mathrm{GHz}$ and $0.63 \mathrm{~dB}$ at $5.5 \mathrm{GHz}$.

\section{2) Configuration 2: Contacts on both sides of the flex line}

In this configuration, the FH34SRJ-8S connector (Hirose ${ }^{\mathrm{TM}}$ ) was applied, considering its 8 contacts on both sides (top and bottom) for the flex line inserted inside.

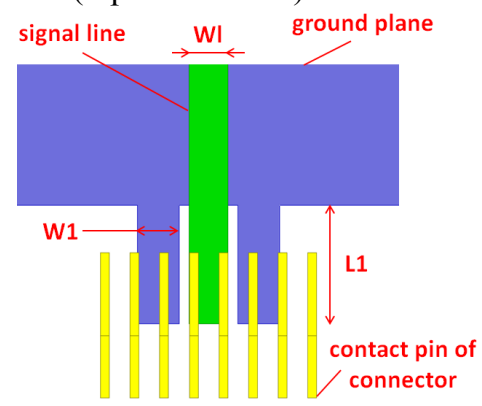

Flexible line side (top view only)

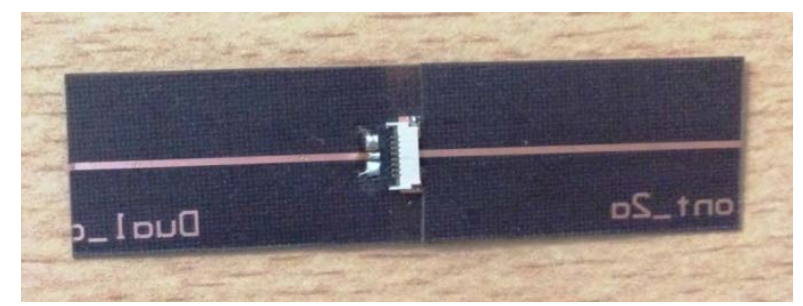

Figure 6: Design for the interconnection in the case of a connector with contacts on both sides of the flex line

For this configuration, the measured insertion losses through the connector are about $0.43 \mathrm{~dB} @ 2.4 \mathrm{GHz}$ and 0.73 $\mathrm{dB} @ 5.5 \mathrm{GHz}$. The results will be detailed during the conference.

\section{INTERCONNECTION FROM ANTENNA TO A MAIN PCB}

In order to prepare the final demonstrator, we have tested the transition from one antenna on a flexible substrate to a feed line on a main PCB. The antenna screen-printed on E4D-200 paper was inserted into a ZIF connector (FH34SRJ-8S), previously soldered on the Teflon ${ }^{\mathrm{TM}}$ substrate for interconnection with the $50 \Omega$ microstrip transmission line, as illustrated in figure 7. Two kinds of antenna (a monopole and a dipole) screen-printed on E4D-200 paper have been tested, with two situations: antenna only and antenna stuck on a small piece of ABS plastic $(2.7 \mathrm{~mm} \times 4.7 \mathrm{~mm} \times 2.55 \mathrm{~mm})$. A simulation model was also realized on HFSS ${ }^{\mathrm{TM}}$ to compare with the measurement.

A reasonably good accordance is obtained between the measurement and the simulation of $S_{11}$ parameters in both cases, despite the ABS was not considered during the antenna design process. It can be consequently compensated easily.
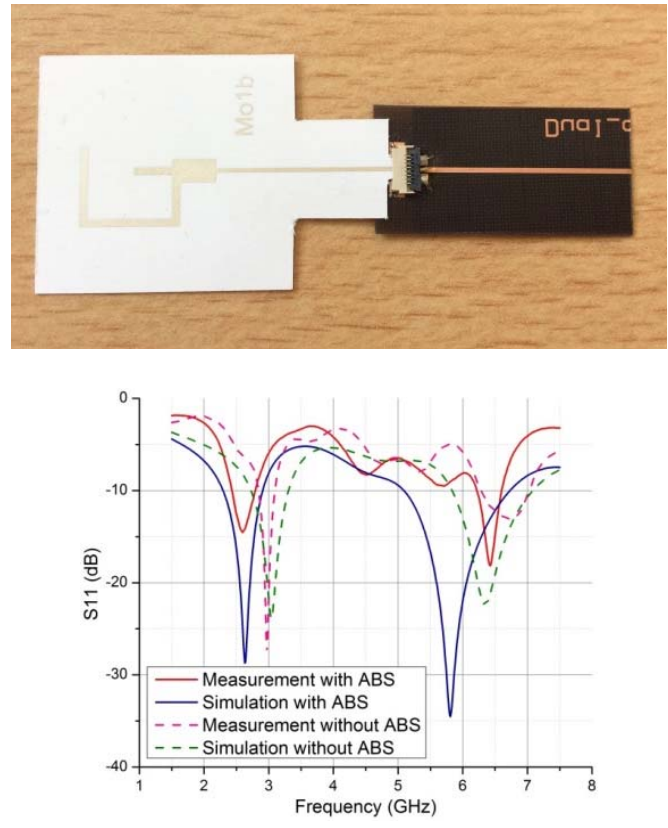

Fig. $7 \mathrm{~S}_{\mathrm{ij}}$ parameters of the transmission line through ZIF connector having contacts on top side of the flex line

\section{CONCLUSION}

We have demonstrated that ZIF connectors can be considered as an innovative solution for flex to PCB interconnections, resolving the use of expensive coaxial cables not appropriate with electronic consumers markets.

Experimentations have been performed on several transmission lines and basic antennas to validate the expected performances

Insertion losses through ZIF connector remain quite small and acceptable, at least up to $6 \mathrm{GHz}$ (about $-0.4 \mathrm{~dB}$ at $2.4 \mathrm{GHz}$ and $-0.7 \mathrm{~dB}$ at $5.5 \mathrm{GHz}$ ).

During the conference, we will present the complete experimental and simulation results concerning both transmission lines and antennas performed on paper substrates.

The next issue of the work will concern a final demonstrator using spatially distributed antennas for radiation patterns and consequently special diversity and multi-standards capabilities.

\section{ACKNOWLEDGMENT}

This research is a part of STICK'IT ANR project in collaboration with INPG Grenoble, and the Paper Technical Center (Grenoble CTP) and Technicolor connected Home Inc. The authors would like to acknowledge the support of Agence Nationale de la Recherche for financial support under grant ANR-IG-CE26-0033-01.

\section{REFERENCES}

[1] H. D. Nguyen et al, "RF characterization of flexible substrates for new conformable antenna systems", 10th European Conference on Antennas and Propagation, 2016.

[2] http://www.webctp.com/ Information on E4D paper

[3] http://www.hirose-connectors.com: Datasheet of FH34 family's 